\title{
Topology Variety Model for Mobile Ad Hoc Networks
}

\author{
Guangli Tian \\ School of Computer Science, \\ Northwestern Polytechnical, \\ University, Xi'an, China \\ guanglitian@gmail.com
}

\author{
Wandong Cai \\ School of Computer Science, \\ Northwestern Polytechnical, \\ University, Xi'an, China \\ caiwd@nwpu.edu.cn
}

\author{
Wei Wang \\ School of Computer Science, \\ Northwestern Polytechnical, \\ University, Xi'an, China \\ wangweinwpu@gmail.com
}

\begin{abstract}
The performances of the Ad Hoc networks vary rapidly because of the change of topology. How to describe and quantify this dynamic characteristic is the basis of designing and simulating the Ad Hoc network. Describing the move patterns of the nodes is the customary way. This way is called mobility model, which have been found several faults. In this paper, Topology Variety Model (TVM) is proposed to describe the dynamic characteristic from the link layer by means of link duration and connectivity probability and it is realized in the simulator ns- 2 . The simulation results indicate that with appropriate PDF of link duration for scenario, TVM can replace mobility model to simulate Ad Hoc networks. Meanwhile, the relationship between mobility and TVM is studied. The influence of dynamic on network performance is simulated based on TVM.
\end{abstract}

\section{Categories and Subject Descriptors}

C.2.1 [Computer-Communication Networks]: Network

Architecture and Design Wireless Communications

\section{General Terms}

Performance, Design, Experimentation.

\section{Keywords}

Ad Hoc networks, mobility model, link duration, performance evaluation, simulation.

\section{INTRODUCTION}

Mobile Ad Hoc networks promise convenient infrastructure-free communication by self-organized multi-hop links between mobile nodes. The movements of nodes bring the topology variety. Researches show that the network performances are closely related to the ability of network protocol to adapt to this dynamic characteristic[1,2]. Describing and quantifying this dynamic characteristic is the basic work of designing and simulating the Ad Hoc network protocols. The traditional way is using mobility models, which describe nodes moving patterns, to make Ad Hoc network topology change according to real world scenario. Many mobility models have been proposed and analyzed[3,4]. The Random Waypoint (RWP) Model[5,6] is most frequently used in

Permission to make digital or hard copies of all or part of this work for personal or classroom use is granted without fee provided that copies are not made or distributed for profit or commercial advantage and that copies bear this notice and the full citation on the first page. To copy otherwise, or republish, to post on servers or to redistribute to lists, requires prior specific permission and/or a fee.

MOBILWARE 2008, February 13-15, Innsbruck, Austria

Copyright () 2008 ICST 978-1-59593-984-5

DOI 10.4108/ICST.MOBILWARE2008.2805
Ad Hoc networks researches and is implemented in the network simulation tool ns-2 and GloMoSim.

Several shortcomings exist in current mobility models. Firstly, some mobility models have shortcomings in definition, which cause obvious deviation in simulation results[1,7,8,9]. This includes that: model convergence time is too long; stationary distribution of nodes is not uniform; boundary effect can't be avoided and so on. An appropriate mobility model that is easy to calculate without shortcomings above has not been proposed[4].

Secondly, the direct metrics in the mobility models (e.g. velocity) can't accurately capture different characteristics of the models $[3,10,11]$ and often do not directly reflect topology variety, while the latter is believed to be more influential to network performance. The literature[11] defined a set of requirements that the mobility metrics must meet and pointed out the link duration was appropriate. The literatures above suggest that describing the dynamic characteristic of Ad Hoc networks from the link layer is reasonable and a more direct way than mobility model.

The link duration and the connectivity probability of nodes are still hot research areas. The link duration distribution of four mobility models was easy to be gained from simulation [12], but to derive the distribution equation of link duration is very complex (see subsection 2.3). At the same time, the link duration varies widely even all the nodes have the same speed. So it seems impossible to get the network performance under special intensity of mobility by simulation based on mobility models.

This paper makes two main contributions for Ad Hoc networks simulation and research. First, we proposed a new kind of model called Topology Variety Model (TVM), which describes the mobility of Ad Hoc networks by means of link duration and connectivity and was realized in the simulator ns-2. Second, the relationship between mobility and TVM was studied. Meanwhile, we made some significant simulations based on TVM to find the relation between mobility and the performance of network.

\section{TOPOLOGY VARIETY MODEL}

\subsection{Model}

We first focus the random mobility models, which mean the nodes, can move to any destination with random velocities or directions (e.g. RWP model, Random Walk (RW) model and Random Direction (RD) model) and the nodes have the same transmission range.

Since nodes are mobile, links between nodes are set up and torn down dynamically. We assume that a link is either up or down. Two nodes without a link between them will establish such a link as soon as they become aware of each other, e.g., when they come within transmission range of each other and packets from each 
other can be successfully decoded. The threshold distance is devoted by $r_{0}$. Although this is not needed for the analysis, communication links are assumed bidirectional since such bidirectional communication is typically required between two nodes for reliably forwarding packets. Let $N=\left\{N_{1}, \ldots, N_{n}\right\}$ denote the set of $n$ mobile communicating nodes. We model the link between two nodes as follows: For distinct nodes $N_{i}$ and $N_{j}$ in $N$, we introduce a $\{0,1\}$-valued reachability process $\left\{\xi_{i j}(t), t>0\right\}$ with the interpretation that $\xi_{i j}(t)=1$ (resp. $\xi_{i j}(t)=0$ ) if the "link" $(i, j)$ is up (resp. down) at time $t \geq 0$. Since the communication links are assumed bidirectional, we must have $\xi_{i j}(t)=\xi_{j i}(t)$ and $\xi_{i i}(t) \equiv 0$. By introducing a time-varying set $E(t)$ of directed edges through the relation $E(t)=\left\{(i, j) \in N \times N: \xi_{i j}(t)=1, t>0\right\}$, we can devote the topology by a time-varying graph structure $G(t)\langle N, E(t)\rangle$.

The process $\left\{\xi_{i j}(t), t>0\right\}$ is simply an alternating on-off process, with successive up and down time durations given by the rvs $\left\{\ell_{i j}(k), k=1,2, \ldots\right\} \quad$ and $\quad\left\{\gamma_{i j}(k), k=1,2, \ldots\right\} \quad, \quad$ respectively. For $i, j \in N, i<j$, the processes $\xi_{i j}(t)$ are mutually independent and $\ell_{i j}$ (or $\gamma_{i j}$ ) are independent and identically distributed rvs. So we can produce the logical topology $G(t)\langle N, E(t)\rangle$, with the probability density function (PDF) of link duration $\ell$ and linkbroken duration $\gamma$. As the path duration is decided by the link duration [13], and in most scenarios, $E \gamma \gg E \ell$, the PDF of $\gamma$ makes less sense than PDF of $\ell$ to the network performance. To simplify the model, we assume the kind of the PDF of $\gamma$ is the same with PDF of $\ell$ and $f_{t}(\gamma)=f_{t}\left(C_{0} \ell\right)$ with $C_{0}=E \gamma / E \ell$. So the nodes connectivity probability $P_{0}$, which means the probability of that a couple of nodes have direct link, can be devoted by

$$
P_{0}=E \ell /(E \ell+E \gamma)=1 /\left(1+C_{0}\right)
$$

Meanwhile, from the spatial view, $P_{0}$ can be devoted by

$$
P_{0}=r_{0}^{2} / R_{0}^{2}
$$

, where $R_{0}$ means the radius of the deployment region. So the mobility and the connectivity of Ad Hoc networks can be measured by the metrics $\ell$ and $P_{0}$. We illustrate these notions on the seven nodes situation depicted in Figure 1 . The duration of symbol ' 1 ' in matrix means link duration $\ell$ and the ratio of symbol ' 1 ' means the connectivity $P_{0}$.

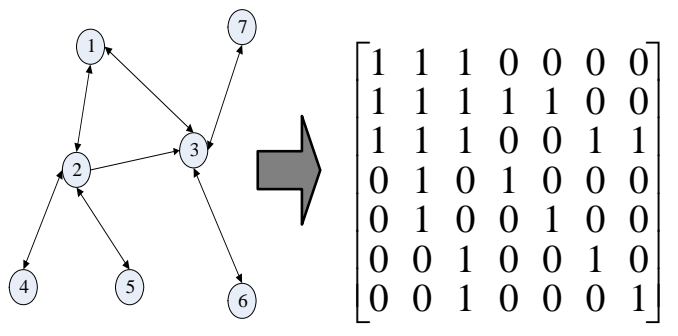

Figure 1. An example of topology graph at a moment.
Reversely, if we know the PDF of link duration and the connectivity of nodes (just as we know the mobility pattern and the speed of the node), how to find to produce the varied topology $G(t)\langle N, E(t)\rangle$ is the function of TVM.

\subsection{Link Duration}

The PDF of link duration can be obtained from three ways. First, PDF of link duration is derived from mobility model. Different mobility patterns lead to different link durations. According to the movement rules of mobile nodes, we can get the relative positions and speeds. Then the PDF of link duration can be derived as shown in section 2.3. If movement of nodes has spatial dependence and temporal dependence, (such as Freeway Mobility (FM) model) we only use the following approach.

Secondly, we can get the statistical PDF of link duration by polynomial fit, based on analysis and statistics on the output file of mobility model program (such as setdest in ns-2). We used a similar approach as [12], and developed an analytical tool named pstn2lnk. The format of input file should be compatible with the format required by ns-2, like the file generated by setdest and mobility-generator in [14].

Thirdly, the PDF of link duration is set directly for some special purpose. For example, we can define a suitable PDF (such as $\Gamma$ distribution) of link duration, which can generally cover most common movement patterns, to replace lots of mobility models. This will not only be useful for simulation, but also will provide a standard for comparison capability among different network protocols. Many papers present new routing protocols using different mobility models or with different metrics in simulation environment, without a uniform standard, it is usually unable to tell which is better. Another application is giving a set of constant values for the link duration to get adapting capability of network protocol for the different dynamic intensity (shown in section 3.2).

\subsection{Link duration of random mobility models}

For random mobility models, including RWD, RW, RD models, link duration can be expressed approximately by one complicated distribution with some assumptions. We assume the pause time is zero and $R_{0} \gg r_{0}$. The cumulative distribution function (CDF) of link duration of any two nodes (credited to $n_{1}$ and $n_{2}$ ) can be devoted by

$$
F_{t}(t)=\iint F_{t}\left(\ell_{12} \mid v_{1}, v_{2}\right) f_{v}\left(v_{1}\right) f_{v}\left(v_{2}\right) d v_{1} d v_{2}
$$

, where the $v_{1}$ and $v_{2}$ devote the velocities of $n_{1}$ and $n_{2}$. The PDF of velocity of RWP model is $f_{v}(v)=\frac{1}{v}\left(\ln \frac{v_{\max }}{v_{\min }}\right)^{-1}$, $f_{v}(v)=\frac{1}{v}\left(\ln \frac{v_{\max }}{v_{\min }}\right)^{-1}, \quad v \in\left[v_{\min }, v_{\max }\right]$. The conditional CDF of link duration of RWP model is given by Lemma 1 and Lemma 2.

The relative speed of node $n_{2}$ with respect to node $n_{1}$ is denoted by $\vec{v}$, then $\vec{v}=\vec{v}_{2}-\vec{v}_{1}$. The directions of the nodes are denoted by $\theta_{v 1}, \theta_{v 2}$ and $\theta_{v}$, separately. We set $\theta_{v 1}=0$ and $v_{1}=k v_{2}$, then $\theta_{v 2}=\varphi, f\left(\theta_{v 2}\right)=U(0,2 \pi)$

Lemma 1. If $v_{1}>v_{2}$, then 


$$
\begin{aligned}
& F_{t}\left(\ell_{12} \mid v_{1}>v_{2}\right)= \\
& \int_{0}^{\frac{\pi}{2}} \int_{\theta_{v 2} \in \Psi} \sqrt{k^{2}+1-2 k \cos \theta_{v 2}} \sin \left(\frac{\pi}{2}-\theta-\arcsin \frac{\sin \theta_{v 2}}{\sqrt{k^{2}+1-2 k \cos \theta_{v 2}}}\right) d \theta_{v 2} d \theta \\
& \int_{0}^{\frac{\pi}{2}} \int_{\theta_{v 2} \in \Theta} \sqrt{k^{2}+1-2 k \cos \theta_{v 2}} \sin \left(\frac{\pi}{2}-\theta-\arcsin \frac{\sin \theta_{v 2}}{\sqrt{k^{2}+1-2 k \cos \theta_{v 2}}}\right) d \theta_{v 2} d \theta \\
& \text { with } \Theta=\left\{\begin{array}{ll}
{[0, \theta-\arccos (k \cos \theta)) \cup(\theta+\arccos (k \cos \theta), 2 \pi)} & \text { if } k \cos \theta<1 \\
{[0,2 \pi)} & \text { if } k \cos \theta \geq 1
\end{array}\right. \text {, } \\
& \Psi=\left\{\theta_{v 2} \mid \frac{2 r_{0} \cos \left(\theta+\arcsin \frac{\sin \theta_{v 2}}{\sqrt{k^{2}+1-2 k \cos \theta_{v 2}}}\right)}{v_{2} \sqrt{k^{2}+1-2 k \cos \theta_{v 2}}}<t, \theta_{v 2} \in \Theta, \theta \in\left[0, \frac{\pi}{2}\right)\right\} .
\end{aligned}
$$

The proof of Lemma 1 is shown in Appendix.

Lemma 2. If $v_{1}<v_{2}$, then

$$
\begin{aligned}
& F_{t}\left(\ell_{12} \mid v_{1}<v_{2}\right)= \\
& \frac{\int_{0}^{\frac{\pi}{2}} \int_{\theta_{v 2} \in \Psi_{11}} A B d \theta_{v 2} d \theta+\int_{0}^{\frac{\pi}{2}} \int_{\theta_{v 2} \in \Psi_{12}} A C d \theta_{v 2} d \theta+\int_{\frac{\pi}{2}}^{\pi} \int_{\theta_{22} \in \Psi_{21}} A C d \theta_{v 2} d \theta+\int_{\frac{\pi}{2}}^{\pi} \int_{\theta_{v 2} \Psi_{22}} A B d \theta_{v 2} d \theta}{\int_{0}^{\frac{\pi}{2}} \int_{\theta_{v 2} \in \Theta_{11}} A B d \theta_{v 2} d \theta+\int_{0}^{\frac{\pi}{2}} \int_{\theta_{v 2} \in \Theta_{12}} A C d \theta_{v 2} d \theta+\int_{\frac{\pi}{2}}^{\pi} \int_{\theta_{v 2} \in \Theta_{21}} A C d \theta_{v 2} d \theta+\int_{\frac{\pi}{2}}^{\pi} \int_{\theta_{v 2} \in \Theta_{22}} A B d \theta_{v 2} d \theta} \\
& \text {, with } \Theta_{11}=(\arccos (k \cos \theta)+\theta, 2 \pi-\arccos k), \\
& \Theta_{12}=(2 \pi-\arccos k, 2 \pi+\theta-\arccos (k \cos \theta)), \\
& \Theta_{21}=(0, \arccos (k \cos (\pi-\theta))+\theta-\pi) \cup(2 \pi-\arccos k, 2 \pi), \\
& \Theta_{22}=(\pi+\theta-\arccos (k \cos (\pi-\theta)), 2 \pi-\arccos k), \\
& \Psi_{11}=\left\{\theta_{v 2} \mid D<t, \theta_{v 2} \in \Theta_{11}, \theta \in[0, \pi / 2)\right\}, \\
& \Psi_{12}=\left\{\theta_{v 2} \mid E<t, \theta_{v 2} \in \Theta_{12}, \theta \in[0, \pi / 2)\right\}, \\
& \Psi_{21}=\left\{\theta_{v 2} \mid E<t, \theta_{v 2} \in \Theta_{21}, \theta \in[\pi / 2, \pi)\right\}, \\
& \Psi_{22}=\left\{\theta_{v 2} \mid D<t, \theta_{v 2} \in \Theta_{22}, \theta \in[\pi / 2, \pi)\right\}, A=\sqrt{k^{2}+1-2 k \cos \theta_{v 2},} \\
& B=\sin \left(\pi / 2-\theta-\arcsin \left(\sin \theta_{v 2} / A\right)\right), \\
& C=\sin \left(3 \pi / 2-\theta+\arcsin \left(\sin \theta_{v 2} / A\right)\right), \\
& D=2 r_{0} \cos \left(\theta+\arcsin \left(\sin \theta_{v 2} / A\right)\right) /\left(v_{2} A\right), \\
& E=2 r_{0} \cos \left(\pi-\theta+\arcsin \left(\sin \theta_{v 2} / A\right) /\left(v_{2} A\right) .\right.
\end{aligned}
$$

The simulations run on a rectangular region of $2500 \mathrm{~m} \times 2500 \mathrm{~m}$. There were 40 nodes moving across this region without a pause, and $r_{0}=250 \mathrm{~m}$. Each simulation lasted 2000s, but we only researched the last 1800s in order to reduce the effect of the transient period. We took the average of 5 runs.

The result is shown in Figure 2 and Figure 3. Lines represent the theoretical values, and " $\times$ " represents the experimental values. When the velocity of nodes is larger, experimental and theoretical results show good agreement. When the velocity is smaller, there is a slight difference. The main reason is that the assumption $R_{0} \gg r_{0}$ is not satisfied strictly (in fact, $R_{0}=10 r_{0}$ ), so the probability of speed changing when two nodes have link between them can not be ignored while the velocity is smaller.

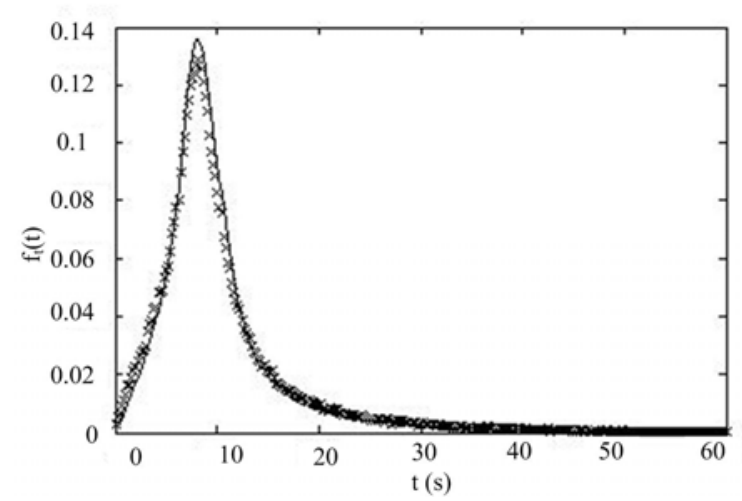

Figure 2. The PDF of link duration with $v_{\min }=20 \mathrm{~m} / \mathrm{s}$, $v_{\max }=40 \mathrm{~m} / \mathrm{s}$.

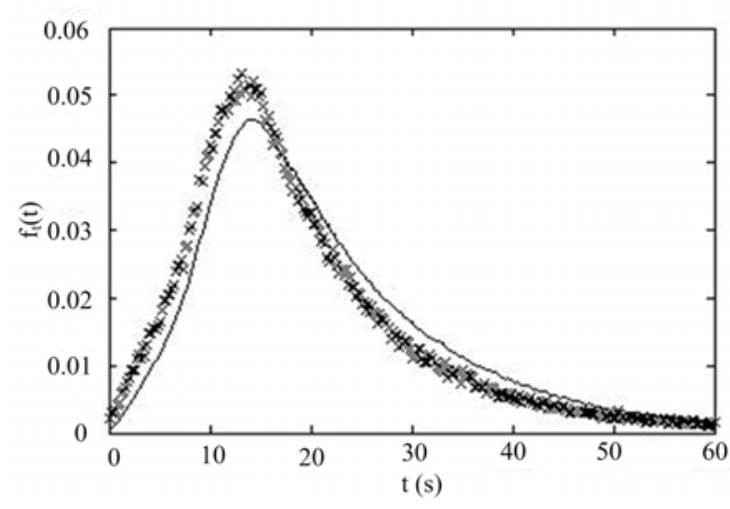

Figure 3. The PDF of link duration with $v_{\min }=5 \mathrm{~m} / \mathrm{s}$,

$$
v_{\max }=30 \mathrm{~m} / \mathrm{s} \text {. }
$$

\subsection{The Implementation of TVM}

To maintain the structure and compatibility of ns-2, first, we developed a similar tool named setlink to generate the scenario file with the same format of setdest. Second, a vector was added to the class of MobileNodeClass as a member variable to record the link states. When a node sends data, the class of Channel controls which nodes can receive the data according to the vector in sending node. Third, we added a global variable to decide using mobility model or TVM in simulation. As we retained all the former functions in ns-2, users can make simulation based on mobility model or TVM only by setting a different value to the global variable in tcl-script.

The input metrics of tool setlink include the number of node (NN), the first number of index (I), the probability of connectivity (P), simulation time (ST), the kind of PDF of link duration (F) and the metrics of PDF (X1,X2 ...). The format of the output scenario file is as follows:

\$ns_at T "\$node_( $\left.\mathrm{N}_{1}\right)$ setlink $\mathrm{N}_{2} \mathrm{~S}$ ".

The core algorithm of setlink is shown in Figure 4. 


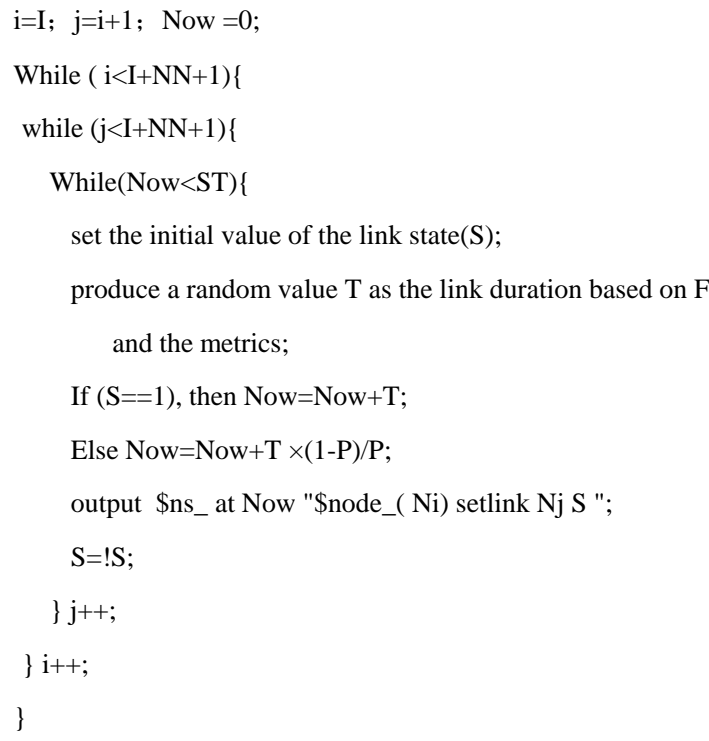

Figure 4. The core algorithm of setlink.

When the nodes in Ad Hoc networks are not equivalent (e.g. the group leader and the common node in Reference Point Group Mobility (RPGM) model,), the scenario files should be generated separately with different input metrics and then merged into the final file with the index of node set appropriately.

\section{SIMULATION BASED ON TVM}

\subsection{Simulation for Different Scenarios}

Mobility models and TVM have different advantages each to simulate scenarios. It is more directly and clearer to use node speed, movement pattern and the region in mobility model. However, it is heavy work to develop software for every mobility model in simulation tool. On the other hand, every scenario can be simulated theoretically based on TVM with appropriate PDF of link duration and simulation result is similar as mobility model under the same dynamic intensity. The below table shows the performances comparison results of three different types of scenario simulated based on the mobility models and TVM with the corresponding metrics.

The simulator was ns-2. The IEEE 802.11 DCF and AODV were used as the MAC protocol and routing protocol. The network data flows were produced by the tool cbrgen. The first mobility model was RWP model. And the connectivity and the link duration were computed by equation (2) and (3). The main metrics in simulation were as follows: $v_{\min }=5 \mathrm{~m} / \mathrm{s}, v_{\max }=30 \mathrm{~m} / \mathrm{s}, \quad N N=40$, $l=2500 \mathrm{~m}, r_{0}=250 \mathrm{~m}$, Packet size $=64$ Byte, interval $=0.25 \mathrm{~s}$. The second mobility model and the third mobility model were FM and RPGM models. The scenario files were generated by mobility-generator. The PDF of link durations were gotten by pstn2lnk.

The move pattern of group leader was generated from RWP model. The distance between the group node and the group leader were less than transmission range, so the connectivity probability between them was 1 . The other main metrics in simulation were as follows: for group leader, $v_{\min }=5 \mathrm{~m} / \mathrm{s}, v_{\max }=50 \mathrm{~m} / \mathrm{s}$, group number $=4$, Node number per group $=10, l=1000 \mathrm{~m}, r_{0}=250 \mathrm{~m}$, packet size $=256$ Byte, interval $=0.25 \mathrm{~s}$, speed deviation ratio $=0.1$, angle deviation ratio $=0.1$. Each simulation lasted $10000 \mathrm{~s}$. All the performances were the average values of 5 runs for all the nodes.

Table 1. The network performance of simulation based on mobility model (MM) and TVM.

\begin{tabular}{|c|c|c|c|c|c|c|}
\hline \multirow{3}{*}{ Scenario } & \multicolumn{6}{|c|}{ Network performance } \\
\hline & \multicolumn{2}{|c|}{$\begin{array}{l}\text { End-to-end } \\
\text { delay(s) }\end{array}$} & \multicolumn{2}{|c|}{ Throughput (K) } & \multicolumn{2}{|c|}{ Loss (\%) } \\
\hline & MM & TVM & MM & TVM & MM & TVM \\
\hline RWP & 1.64 & 1.72 & 3.06 & 3.32 & 70.8 & 72.5 \\
\hline FM & 2.35 & 2.17 & 2.86 & 2.98 & 76.4 & 72.1 \\
\hline RPGM & 0.07 & 0.11 & 63.3 & 60.7 & 1.94 & 2.12 \\
\hline
\end{tabular}

\subsection{Setting static topology}

One of advantages of TVM is that it is every easy to set the ad hoc topology. Sometimes, we have to design the topology of ad hoc network to be some special structure we want, and make simulation to validate something. This means the topology of the network does not vary randomly. The topology of the network is decided by relative distance and the transmission range of nodes. So it is a hard work to compute the right locations of nodes if the number of the nodes is large. By the command setlink, we can set any topology to be we want and change the topology with time easily.

\subsection{Network Protocol and Dynamic Intensity}

Another of advantages of TVM is that we can set the special dynamic intensity of the ad hoc in simulations. One network protocol can't always behave better with different dynamic intensity and mobility pattern [1]. It is very necessary to get the relation between the network performance and the dynamic intensity to design better network protocol. But at the same time, the link duration varies widely even all the nodes have the same speed. So the simulation result is mixed. It seems impossible to get the network performance under special intensity of mobility by simulation based on mobility models.

We make relationship of routing protocol and dynamic intensity for example. In our simulations below, the link durations were a series of constants. The results are significant for routing protocol research. Meanwhile, the result indicates the smaller link durations make more sense than the bigger ones to the performances. For example, the mean performance with the link durations set at 5 s and at $15 \mathrm{~s}$, is worse than the performance with the link duration set at 10s. This conclusion is coincident with the relation of link duration and path duration [13].

The main metrics in simulation were as follows:, $P=0.40$, $N N=40, S T=2000 \mathrm{~s}$. The network data flows were produced by the tool cbrgen and Packet size $=64$ Byte, interval $=0.25 \mathrm{~s}$. All the performances were the average values of 5 runs for all the nodes. 


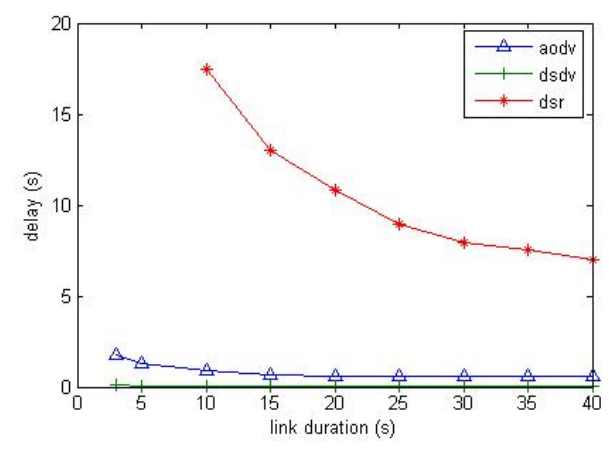

Figure 5. End-to-end delay vs. link duration.

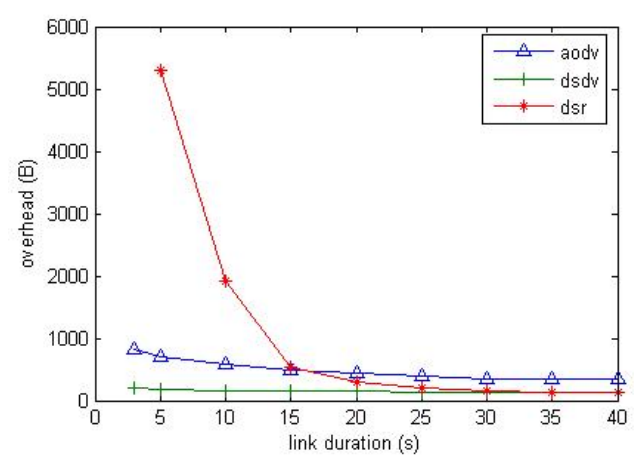

Figure 6. Overhead vs. link duration.

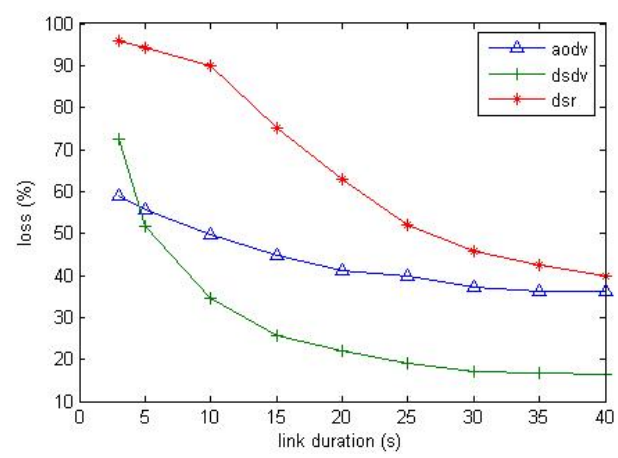

Figure 7. Loss vs. link duration.

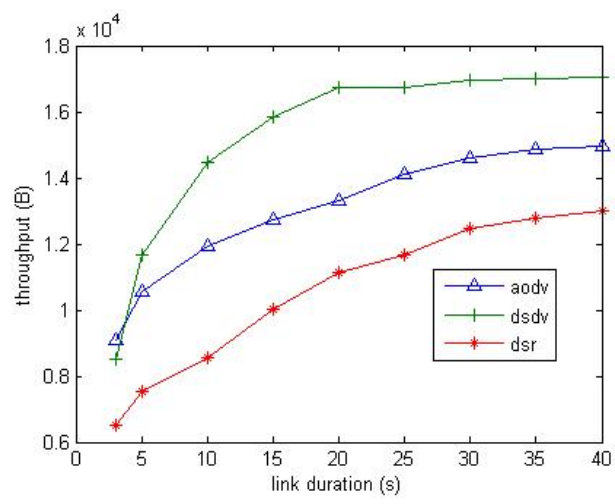

Figure 8. Throughput vs. link duration.

\section{CONCLUSIONS}

In this paper, TVM is defined based on the link layer and is realized in the tool ns-2. And with appropriate PDF of link duration for scenarios, the simulation result is similar for TVM and mobility model. The performance characteristic with different size of link duration is concluded based on the simulation results. Meanwhile, the PDF of link duration of random mobility model is derived. The results seem to be useful for Ad hoc network theoretical research and simulation technology.

\section{REFERENCES}

[1] F. Bai, N. Sadagopan, A. Helmy, "Important: a framework to systematically analyze the impact of mobility on performance of routing protocols for ad hoc networks," In Proc. IEEE computer INFOCOM 2003, San Francisco, 2003, PP. 825-835.

[2] Bhatt M., Chokshi R., Desai S., Panichpapiboon S., Wisitpongphan N., Tonguz O. K, "Impact of mobility on the performance of ad hoc wireless networks,” In Proc TVTC2003-FallT, Orlando, 2003, PP. 3025-3029

[3] Q. Zhang, X. Hong, S. Ray, "Recent advances in mobility modeling for mobile ad hoc," In Proc ACM the 42nd annual Southeast regional conference, Alabama, pp. 70-75, 2004.

[4] Camp, T., Boleng, J. and Davies, V., "A Survey of Mobility Models for Ad Hoc Network Research,” Wireless Communications \& Mobile Computing: Special issue on Mobile Ad Hoc Networking: Research, vol. 2, no. 5, pp. 483-502, 2002.

[5] Johnson, D., and Maltz D., "Dynamic source routing in ad hoc wireless networks,” Mobile Computing, T. Imelinsky and H. Korth, eds, Kluwer Academic Publishers, pp. 153181, 1996.

[6] Broch, J., Maltz D., Johnson, D., Hu, Y., and Jetcheva J., "Multi-Hop wireless ad hoc network routing protocols," In Proc. MOBICOM ’98, Dallas, pp. 85-97, 1998.

[7] J. Yoon, M. Liu, B. Noble, "Random waypoint considered harmful,” In Proc. IEEE INFOCOM 2003, San Francisco, pp. 1312-1321, 2003.

[8] G. Lin, G. Noubir and R. Rajaraman, "Mobility Models for Ad Hoc Network Simulation,” In Proc IEEE INFOCOM 2004, Hong Kong, pp. 454-463, 2004.

[9] Navidi W., Camp T., "Stationary Distributions for the Random Waypoint Mobility Model,” IEEE Transactions on Mobile Computing, vol. 3, no. 1, pp. 99-108, 2004.

[10] Boleng, T. Camp, W. Navidi, "Metrics to enable adaptive protocols for mobile ad hoc networks,” In Proc ICWN 2002, pp. 293-298, 2002.

[11] X. P. Costa, C. Bettstetter, H. Hartenstein, “Toward a mobility metric for comparable \& reproducible results in Ad Hoc networks research,” Mobile Computing and Communications Review, vol. 7, no. 4, pp. 58-60, 2003.

[12] N. Sadagopan, F. Bai, B. Krishnamachari, A. Helmy, "Paths: analysis of path duration statistics and their impact on 
reactive MANET routing protocols,” In Proc MobiHoc'03, Annapolis, pp. 245-256, 2003.

[13] Y. Han, R. La, A. Makowski, S. Lee, "Distribution of path durations in mobile ad-hoc networks-Palm's Theorem to the rescue,” Computer Networks, vol. 50, no. 12, pp. 18871900, 2006,

[14] http://nile.usc.edu/important/software.htm

\section{APPENDIX}

The point, at which the link between $n_{2}$ and $n_{1}$ become up, is denoted by $\left(r_{0}, \theta\right)$. The position relation of $n_{1}$ and $n_{2}$ is shown in Figure 9, where $\theta_{v} \in(\pi / 2+\theta, 3 \pi / 2+\theta)$. As $v_{1}>v_{2}$, the point $\left(r_{0}, \theta\right)$ can only be on the face-half of transmission circle of $n_{1}$. We only need to consider the situation of $\theta \in[0, \pi / 2)$, as it is axial symmetric. The link duration equals the time spent by $n_{2}$ to traverse the transmission circle with speed $v$. The distance is denoted by $l$, and then $\ell_{12}=l / v$. As shown in Figure 9, we can get $l=2 r_{0} \cos (\theta+\beta)$ or $l=2 r_{0} \cos \left(\theta-\beta^{\prime}\right)$. Figure 10 shows the relations of $\vec{v}_{1}, \vec{v}_{2}$ and $\vec{v}$. Then we can get $\theta_{v}=\pi-\beta=\pi-\arcsin \frac{\sin \theta_{v 2}}{\sqrt{k^{2}+1-2 k \cos \theta_{v 2}}}$

and $v=v_{2} \sqrt{k^{2}+1-2 k \cos \theta_{v 2}}$. The range of $\theta_{v 2}$, in which $n_{2}$ can build link with $n_{1}$, is denoted by $\Theta$, and then

$$
\Theta= \begin{cases}{[0, \theta-\alpha) \cup(\theta+\alpha, 2 \pi)} & \text { if } k \cos \theta<1 \\ {[0,2 \pi)} & \text { if } k \cos \theta \geq 1\end{cases}
$$

, where $\alpha=\arccos (k \cos \theta)$. If he range of $\theta_{v 2}$ in $\Theta$ is denoted by $\Psi$ and $\ell_{12}<t$, then

$$
\Psi=\left\{\theta_{v 2} \mid \frac{2 r_{0} \cos \left(\theta+\arcsin \frac{\sin \theta_{v 2}}{\sqrt{k^{2}+1-2 k \cos \theta_{v 2}}}\right)}{v_{2} \sqrt{k^{2}+1-2 k \cos \theta_{v 2}}}<t, \theta_{v 2} \in \Theta, \theta \in\left[0, \frac{\pi}{2}\right)\right\} .
$$

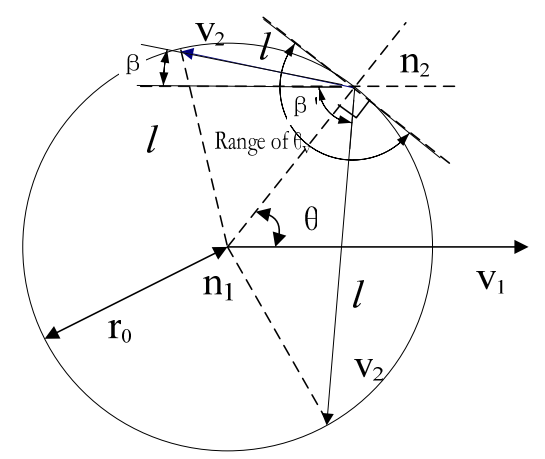

Figure 9.The distance for $n_{2}$ to traverse the transmission circle of $n_{1}$.

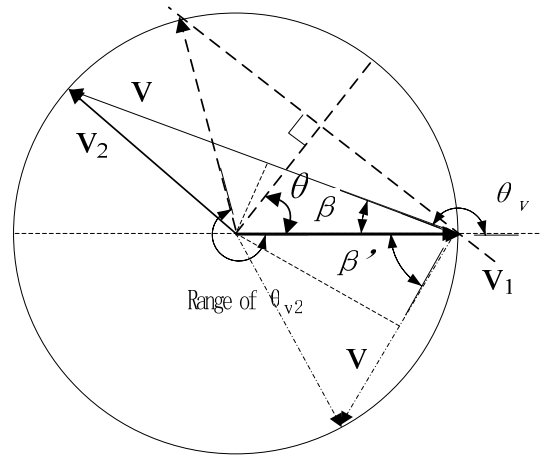

Figure 10. The relation of $\vec{v}_{1}, \vec{v}_{2}$ and $\vec{v}$, when $k>1$.

During the simulation time, devoted by $T_{\text {run }}$, the link between $N_{1}$ в and $N_{2 \mathrm{~B}}$ become up for $m_{12}$ times, and for $\theta \in(t, t+\Delta \theta)$, $\theta_{v 2} \in\left(s, s+\Delta \theta_{v 2}\right), t \in[0, \pi / 2), s \in \Theta$, the times is $\Delta m$. As shown in Figure 11, $\Delta m$ equals to the product of the density of $n \mathrm{~B}_{2 \mathrm{~B}}$ and the acreage (devoted by $S$ ) covered by $\Delta d$ with speed $v$ during $T_{\text {run }}$ :

$$
\begin{aligned}
& \Delta m=\frac{\Delta \theta_{v 2}}{2 \pi} \cdot \frac{S}{\|R\|} \\
& \underset{\substack{\Delta \theta \rightarrow 0 \\
\Delta \theta_{v 2} \rightarrow 0}}{=} \frac{1}{2 \pi\|R\|} r_{0} v T_{r u n} \Delta \theta_{v 2} \Delta \theta \sin \left(\theta_{v}-\theta-\frac{\pi}{2}\right)
\end{aligned}
$$

So the conditional CDF of link duration is

$$
\begin{aligned}
& F_{t}\left(\ell_{12} \mid v_{1}>v_{2}\right)
\end{aligned}
$$

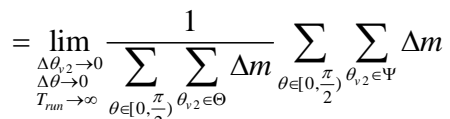

After changing the form, we get

$$
\begin{aligned}
& F_{t}\left(\ell_{12} \mid v_{1}>v_{2}\right) \\
& =\frac{\int_{0}^{\frac{\pi}{2}} \int_{\theta_{v 2} \in \Psi} \sqrt{k^{2}+1-2 k \cos \theta_{v 2}} \sin \left(\frac{\pi}{2}-\theta-\arcsin \frac{\sin \theta_{v 2}}{\sqrt{k^{2}+1-2 k \cos \theta_{v 2}}}\right) d \theta_{v 2} d \theta}{\int_{0}^{\frac{\pi}{2}} \int_{\theta_{v 2} \Theta \Theta} \sqrt{k^{2}+1-2 k \cos \theta_{v 2}} \sin \left(\frac{\pi}{2}-\theta-\arcsin \frac{\sin \theta_{v 2}}{\sqrt{k^{2}+1-2 k \cos \theta_{v 2}}}\right) d \theta_{v 2} d \theta}
\end{aligned}
$$

The result follows.

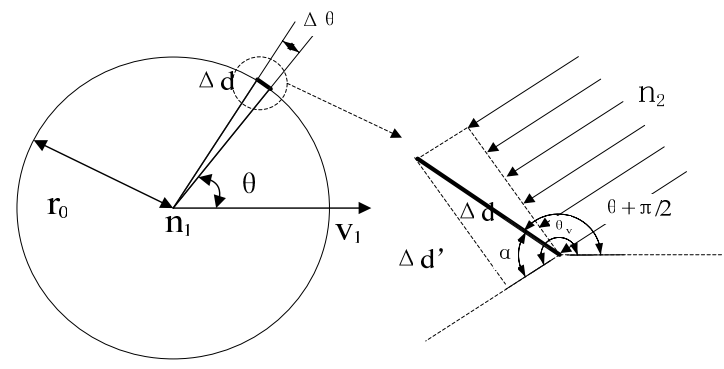

Figure 11. Computing the acreage discovered by $\Delta d$. 